\title{
0 $2 \pi$ \\ Indicador de Pobreza Multidimensional como síntese dos efeitos da abordagem multissetorial do Plano Brasil Sem Miséria
}

Alexander Cambraia N. Vaz Paulo de Martino Jannuzzi

1 Doutor em Ciência Política (UFMG). Consultor externo da FAO, Organização das Nações Unidas para Alimentação e Agricultura.

2 Doutor em Demografia (UNICAMP), Professor da ENCE/IBGE, Secretário de Avaliação e Gestão da Informação do Ministério de Desenvolvimento Social e Combate à Fome. 


\section{Resumo}

Pobreza É um fEnÔMENo MULTIDIMENSIONAL E MUlTIFACETADO. MULTIDIMENSIONAL PELA NATUREZA COMPLEXA DE SUA CARACTERIZAÇÃO E DIMENSIONAMENTO; MULTIFACETADA PELA DIVERSIDADE DE PÚBLICOS E FORMAS COMO ELA SE MANIFESTA. A FOME, A DESNUTRIÇÃO, A INSERÇÃO PRECÁRIA DO MERCADO DE TRABALHO, A BAIXA RENDA, A MORADIA INADEOUADA, A DIFICULDADE DE ACESSO A POSTOS DE SAÚDE OU ESCOLAS, A INEXISTÊNCIA DE SERVIÇOS DE INFRAESTRUTURA URBANA, O PRECONCEITO DE RAÇA E GÊNERO REVELAM DIMENSÕES ESPECÍFICAS DE VULNERABILIDADE E POBREZA OUE PODEM ACOMETER CONTINGENTES MAIORES OU MENORES DA POPULAÇÃO. COM TAL NATUREZA MULTIDIMENSIONAL E MULTIFACETADA, A POBREZA REQUUER ESTRATÉGIAS MULTISSETORIAIS DE ATUAÇÃO, INSPIRADAS EM MODELOS MULTIDISCIPLINARES DE INTERVENÇÃO PARA SUA MITIGAÇÃO E EFETIVA SUPERAÇÃO. ESSE TEM SIDO UM PRESSUPOSTO BÁSICO NA FORMULAÇÃO DE AÇÕES E PROGRAMAS DAS Políticas de Desenvolvimento Social e COMBATE À FOME AO LONGO DOS ÚLTIMOS DOZE ANOS, E PRINCÍPIO ORGANIZATIVO MARCANTE DO Plano BRAsil Sem MisérIA (BSM) DESDE 2011. ESSA ABORDAGEM COMPLEXA DE INTERVENÇÃO REQUER UM CONJUNTO AMPLO DE PESOUISAS E INDICADORES PARA MONITORAMENTO DOS AVANÇOS E DIFICULDADES DE SUAS AÇÕES E PROGRAMAS E TAMBÉM DOS SEUS RESULTADOS E IMPACTOS. TRAZ-SE NESSE ARTIGO A PROPOSTA DE UMA MEDIDA SÍNTESE DOS EFEITOS DO PLANO SOBRE A POPULAÇÃO BRASILEIRA, A PARTIR DA PROPOSTA DE EXPERIÊNCIAS INTERNACIONAIS DE INDICADORES DE POBREZA MULTIDIMENSIONAL.

\section{Abstract}

POVERTY is A MULTIDIMENSIONAL AND MULTIFACETED PHENOMENON. MULTIDIMENSIONAL BY THE COMPLEX NATURE OF ITS CHARACTERIZATION AND SIZING; BY PUBLIC MULTIFACETED DIVERSITY AND WAYS IT MANIFESTS ITSELF. HUNGER, MALNUTRITION, POOR INTEGRATION OF THE LABOR MARKET, LOW INCOME, POOR HOUSING, POOR ACCESS TO HEALTH CENTERS OR SCHOOLS, THE LACK OF URBAN INFRASTRUCTURE SERVICES, PREJUDICE OF RACE AND GENDER REVEAL SPECIFIC DIMENSIONS VULNERABILITY AND POVERTY THAT CAN AFFECT LARGER OR SMALLER CONTINGENT OF THE POPULATION. WITH SUCH A MULTIDIMENSIONAL AND MULTIFACETED NATURE, POVERTY REQUIRES MULTISECTORAL STRATEGIES OF ACTION, INSPIRED BY MULTIDISCIPLINARY INTERVENTION MODELS FOR THEIR MITIGATION AND EFFECTIVE REPLACEMENT. THIS HAS BEEN A BASIC ASSUMPTION IN THE FORMULATION OF actions and programs of the Social Policy Development and Hunger Alleviation over the LASt tWELVE yeARS, AND STRIKING ORGANIZING PRINCIPLE OF BRAZIL WITHOUT POVERTY PLAN (BSM) SINCE 2011. THIS COMPLEX INTERVENTION APPROACH REQUIRES WIDE RANGE OF RESEARCH AND INDICATORS FOR MONITORING PROGRESS AND DIFFICULTIES OF THEIR ACTIONS AND PROGRAMS AND ALSO THE RESULTS AND IMPACTS. BRINGS UP THAT ARTICLE THE PROPOSAL FOR A SUMMARY MEASURE OF THE EFFECTS OF THE PLAN ON THE BRAZILIAN POPULATION, FROM THE MULTIDIMENSIONAL INDICATORS PROPOSED BY INTERNATIONAL ORGANIZATIONS.

\section{PALAVRAS-CHAVE:}

pobreza multidimensional; políticas sociais; intersetorialidade; Banco Mundial

Revista Brasileira de Monitoramento e Avaliação | Número 8 | Julho-Dezembro de 2014

Indicador de Pobreza Multidimensional como síntese dos efeitos da abordagem multissetorial do Plano Brasil Sem Miséria 


\section{Introdução}

Pobreza é um fenômeno multidimensional e multifacetado. Multidimensional pela natureza complexa de sua caracterização e dimensionamento; multifacetada pela diversidade de públicos e formas como ela se manifesta. A fome, a desnutrição, a inserção precária do mercado de trabalho, a baixa renda, a moradia inadequada, a dificuldade de acesso a postos de saúde ou escolas, a inexistência de serviços de infraestrutura urbana, o preconceito de raça e gênero revelam dimensões específicas de vulnerabilidade e pobreza que podem acometer contingentes maiores ou menores da população. Esses contingentes vulneráveis podem se revelar por traços e facetas muito marcantes como os de desempregados sem acesso a seguro-desemprego, subempregados, trabalhadores volantes, mães solteiras com filhos ou idosos sem recursos e sem assistência, população em situação de rua, pequenos agricultores familiares sem acesso a crédito e assistência técnica, comunidades ribeirinhas, indígenas e quilombolas residentes em localidades remotas.

Com tal natureza multidimensional e multifacetada, a pobreza requer estratégias multissetoriais de atuação, inspiradas em modelos multidisciplinares de intervenção para sua mitigação e efetiva superação. Esse tem sido um pressuposto básico na formulação de ações e programas das Políticas de Desenvolvimento Social e Combate à Fome ao longo dos últimos doze anos, e princípio organizativo marcante do Plano Brasil Sem Miséria (BSM) desde 2011. Partindo de um diagnós- tico de pobreza multidimensional - a pobreza se revela por várias carências sociais além da insuficiência de renda -, de pobreza multifacetada - a pobreza se manifesta por uma diversidade de públicos vulneráveis - o BSM tem ampliado o escopo dessa estratégia multissetorial e multidisciplinar de intervenção programática, com a proposição e execução de mais de uma centena de ações, envolvendo intensa articulação em nível federal com mais de dez ministérios e pactuação federativa com estados e municípios.

Essa abordagem complexa de intervenção requer um conjunto amplo de pesquisas e indicadores para monitoramento dos avanços e dificuldades de suas ações e programas e também dos seus resultados e impactos. Desde 2011 há um conjunto amplo de pesquisas e indicadores que vem sendo produzidos com esses propósitos, publicados por diversas instituições ${ }^{3}$, além dos instrumentos de gestão e acompanhamento desenvolvidos pela Secretaria Extraordinária de Superação da Extrema Pobreza e da Secretaria de Avaliação e Gestão da Informação ${ }^{4}$.

Como estratégia complementar a esse conjunto diverso de recursos, que permitem analisar aspectos específicos de ações e programas nas diversas áreas setoriais de atualização do BSM, em diferentes níveis de representação territorial, traz-se nesse artigo a proposta de uma medida síntese dos efeitos do Plano sobre a população brasileira, a partir de propostas de instituições multilaterais. 


\section{A pobreza como fenômeno multidimensional}

Analiticamente, a dificuldade em mensurar a pobreza e estabelecer parâmetros de comparação entre diferentes contextos econômicos remete, antes, à necessidade de delinear um indicador de pobreza absoluta. O acesso aos itens fundamentais para a reprodução material (podendo ter pesos diferenciados entre si) pode estabelecer dois limites: o da pobreza extrema e o da pobreza. Pobreza extrema e pobreza podem ser situadas de maneira relativa, expressando assim o percentual de indivíduos ou domicílios abaixo de seus limites, mensurando a incidência da população nesses dois quesitos. A aparente facilidade trazida à baila através da utilização dessa metodologia, deve ser tratada, não obstante, como facilidade aparente. Na realidade, se pegarmos apenas o caso do Brasil, por exemplo, notamos que, mesmo adotando um padrão conceitual de pobreza focado estritamente na renda, existem dificuldades significativas do ponto de vista metodológico.

Como discutido em Jannuzzi et al (2012), dependendo da fonte de dados utilizada e, em especial, do conceito adotado, obtém-se, inevitavelmente, diferentes quantidades de indivíduos passíveis de classificação como extremamente pobres ${ }^{5}$, em um intervalo de 7 milhões a quase 20 milhões. Essas diferenças observadas nos dados, através de conceitos e fontes distintas, passam a clara mensagem de que a própria condição de pobreza pode variar em grau, intensidade e tipo, especialmente em função de contextos sócio demográficos e subconjuntos populacionais específicos. Isso implica reconhecer que, por exemplo, determinados contextos podem "conviver" com sobreposições de pobreza. A pobreza, portanto, não depende exclusivamente da renda, o que

3 Vide, nesse sentido o livro de balanço de dez anos do Programa Bolsa Família (Campello \& Neri 2013), o Relatório de Acompanhamento de Objetivo de Desenvolvimento do Milênio do IPEA e o Relatório Indicadores de Desenvolvimento Brasileiro, organizado pelo Ministério do Planejamento. Todos eles estão disponíveis no portal SAGl em www.mds.gov.br/sagi -> publicações.

4 Vide balanços anuais do Plano BSM, Portal Brasil Sem Miséria no seu Estado e no seu Município, aplicativo Painel de Indicadores de Conjuntura e Programas Sociais nos sítios das Secretarias, acessíveis pelo Portal www.mds.gov.br .

5 Se compararmos, por exemplo, apenas as pesquisas elaboradas por um mesmo órgão estatístico, que é o IBGE, sendo o Censo Demográfico 2010 e a Pesquisa Nacional por Amostra de Domicílios de 2009, essa lógica fica clara. Ao considerarmos, sem qualquer tipo de filtro, a variável classificadora de renda no Censo 2010, obtemos o total de quase 20 milhões de indivíduos em extrema pobreza. Já no caso da PNAD 2009, considerando a mesma variável, temos o total de pouco mais de 9 milhões de indivíduos nessa situação. Com a diferença, portanto, de apenas 1 ano, temos uma contagem de extremamente pobres significativamente diferente entre ambas as pesquisas.

Revista Brasileira de Monitoramento e Avaliação | Número 8 | Julho-Dezembro de 2014 
requer dos agentes interventores a capacidade de desenhar políticas que efetivamente levem em consideração essas diferenciações e o seu caráter multicausal.

Considerar a pobreza em sua forma estritamente monetária pode não contemplar as suas várias dimensões (que não sejam as relacionadas à renda e ao consumo) e outras formas possíveis de mensuração, sendo necessária a construção de outros instrumentos de análise. Afinal, se os critérios para definição da condição de pobre variaram, também devem variar, sugere-se, as ações e políticas destinadas ao seu tratamento. A cada definição específica da categoria, devem corresponder estratégias diferenciadas de intervenção, especialmente porque elementos diversos são tomados como base da condição de pobre.

Porém, perceber a pobreza como um fenômeno multidimensional inevitavelmente leva a uma série de problemas de cunho verdadeiramente filosófico: quais são as capacidades que, quando ausentes, levam a pessoa a uma situação de vulnerabilidade? Como podemos selecioná-las de forma concisa e sistemática? Que capacidades - quando ausentes - afetam mais diretamente a vida e o bem-estar de um indivíduo? A necessidade de uma abordagem mais ampla sobre a medição da pobreza, contemplando enfoques de perspectivas de direitos básicos, de cidadania e do desenvolvimento humano, vem fomentando a proposição de metodologias que expressem as capacidades ou suas privações.

\section{Aspectos metodológicos da pobreza multidimensional}

A principal questão que norteia o presente trabalho concerne aos desafios imputados à gestão pública advindos da natureza multidimensional e multifacetada da pobreza e as questões expressas acima são capazes de elucidar de maneira significativa as dificuldades envolvidas nesse processo. Segundo Feres e Villatoro (2013), as metodologias para computação de índices de pobreza multidimensional podem ser agrupadas em dois conjuntos:

a) Estratégias empíricas, utilizando-se de técnicas multivariadas: visa reduzir a variabilidade das respostas de um conjunto de indicadores a um número menor de dimensões;

b) Metodologias orientadas por axiomas: índices multidimensionais de um conjunto de propriedades desejáveis.

Os autores afirmam que um indicador de pobreza multidimensional contemplaria três aspectos: 1) seleção das dimensões; 2) definição de padrões mínimos para as dimensões; e 3) agregação das dimensões em um índice. A agregação apresenta relações entre as dimensões, podendo estabelecer que o indivíduo apresenta privação em ao menos uma dimensão, como no índice de necessidades básicas insatisfeitas (NBI) para a América Latina (década de 1980). 
Lidando com uma série de formas de mensuração diferentes, uma importância inicial é estabelecer um padrão de notação que produza resultados comparáveis. Nesse trabalho iremos lidar com duas diferentes formas de mensuração da pobreza nesse sentido, adotadas por duas diferentes instituições. Primeiro, - MPI, atualmente adotado pelo PNUD para comparação internacional da situação dos países em termos de pobreza multidimensional, é um dos exemplos mais recentes nesse sentido. O Índice foi elaborado pela Oxford Poverty \& Human Development Iniciative no âmbito dos chamados Objetivos do Milênio, da Organização das Nações Unidas (ONU), conforme proposta da Reunião de Cúpula do Milênio de eliminação da extrema pobreza de todos os países. Atualmente vem sendo adotado como um indicador capaz de perpassar, sugere-se, a linha de corte monetária como parâmetro unitário de avaliação e definição de suas bases ${ }^{6}$. O segundo índice a ser estudado concerne àquele elaborado pelo Banco Mundial e que vem servindo de referência para adoção de políticas de avaliação e de combate à pobreza no âmbito das ações do Organismo.

\section{- ouAdRO 1: TIPOLOGIA do ÍNDICE DE POBREZA MULTIDIMENSIONAL - OPHI/PNUD (2010)}

\begin{tabular}{lll}
\multicolumn{1}{|c}{ INDICADORES ESPECÍfICOS } & \multicolumn{1}{c|}{ A PESSOA ESTÁ PRIVADA SE: } \\
\hline Anos de escolaridade completados & - & Nenhum membro do domicílio completou cinco anos de ensino \\
\hline Frequência à escola das crianças & - & Pelo menos uma criança em idade escolar (até $8^{\circ}$ ano) não matriculada \\
\hline Nutrição & - & Pelo menos um membro da casa está desnutrido \\
\hline Mortalidade infantil & - & Uma ou mais crianças do domicílio morreram \\
\hline Eletricidade & - & Não há eletricidade \\
\hline Saneamento & - & Não há saneamento adequado \\
\hline Água & - & Não há acesso à água potável \\
\hline Piso & - & Mora em casa com piso de terra \\
\hline Combustível para cozinhar & - & Usa combustível "contaminante" (esterco, lenha ou carvão) para cozinhar \\
\hline Bens & - & Não tem carro, caminhão ou veículo motorizado e possui um dos seguintes \\
\hline
\end{tabular}

6 Sua importância reside justamente na adoção de um viés de mensuração da pobreza que reflita, para além das condições monetárias dos indivíduos e famílias, as condições de vida, em termos de moradia, acesso a bens e serviços, dentre outros elementos, como pontos fundamentais para compreensão do fenômeno.

Revista Brasileira de Monitoramento e Avaliação | Número 8 | Julho-Dezembro de 2014 
O índice estruturado pela OPHI apresenta esse foco metodológico em dimensões não estritamente econômicas do desempenho de um país, propondo uma base de indicadores mais abrangente que a do NBI, ou mesmo, vale dizer, do IDH. Abarca questões que perpassam diversos temas e fatores impactantes na realidade social de um país, como escolaridade, saúde e saneamento básico (Quadro 1). O MPI abrange 104 países, tendo por base microdados de pesquisas domiciliares que abarcam temas relacionados às privações em termos de educação, saúde e qualidade de vida. Seu cálculo tem o indivíduo como unidade de análise. Metodologicamente, a arquitetura do índice compreende dimensões que se desagregam em componentes e posteriormente em indicadores.

Seja $y=\left[y_{i j}\right]_{n x d}$ a matriz de realizações para o i-ésimo individuo e a j-ésima privação, considerando uma população de $\mathrm{n}$ indivíduos e d privações. Uma entrada típica dessa matriz, $y_{i j} \geq 0$, corresponde ao valor atribuído à privação j para o individuo i dentro das d diferentes possibilidades. Temos assim que cada vetor linha $y_{i}=\left(y_{i 1} y_{i 2} \ldots y_{i d}\right)_{1 x d}$ apresenta a distribuição do indivíduo i segundo suas privações enquanto um vetor coluna $y_{j}^{\prime}=\left(y_{1 j} y_{2 j} \ldots\right.$ $\left.y_{n j}\right)_{1 x n}$ avalia a distribuição da privação j para os plausíveis indivíduos. Por meio de um vetor de pesos $w=\left(w_{j}\right)_{1 x d} ; w_{j}>0$, podemos ainda atribuir maior importância comparativa a determinada privação, ou balancear igualmente os pesos dentro de uma dimensão específica de privações (saúde ou educação são exemplos). Usando a matriz y como base, o objetivo é identificar pessoas em situações de vulnerabilidade. Para isso, inicialmente definimos o vetor das linhas de pobreza para cada uma das dimensões de privação consideradas: $z=$ $\left(z_{j}\right)_{1 x d} ; z_{j} \geq 0$. Podemos agora definir a matriz de privações $p=\left[p_{i j}\right]$ nxd ; na qual: $p_{i j}=p_{j}$ caso $y_{i j}<z_{j}$ $e p_{i j}=0$ em caso contrário.

O processo de identificar pessoas em situação de vulnerabilidade e mais especificamente pobres e extremamente pobres em situação de vulnerabilidade é feito partindo da matriz de privações. Seja $c=\left(c_{i}\right)_{1 \times n}$ o vetor de privações, cujo elemento típico tem a forma $\left.c_{i}=\sum_{j}^{d}=1\right)^{\wedge} p_{i i^{\prime}}$ sendo, portanto, a soma dos pesos das atribuídos a cada privação que o i-ésimo indivíduo possui. Considere agora um segundo ponto de corte $k$, os indivíduos multidimensionalmente pobres são aqueles para os quais $c_{i} \geq k$. Dentre esse grupo, identificamos, por fim, o grupo de pessoas em situação de extrema pobreza (renda domiciliar per capita de R\$ 70 ou menos) e daqueles já considerados como não pobres (renda domiciliar per capita de R\$ 140 ou mais) como se observa na Figura 1.

As categorias de pobreza para uma determinada população podem ser sumarizadas (Figura 1). Os severamente pobres constituem a parte da população que é simultaneamente extremamente pobre em renda (abaixo da linha de extrema pobreza) e carente em várias dimensões. Naturalmente, espera-se que quanto maior o número de privações, mais difícil seja de sair dessa condição. Os moderadamente pobres são aqueles já acima da linha da extrema pobreza (mas abaixo da linha de pobreza), porém, ainda em uma situação de vulnerabilidade quando consideradas as privações. Os vulneráveis por escassez, apesar de já se encontrarem numa posição razoável quanto à renda, são considerados multidimensionalmente pobres. 


\section{- FIGURA 1: MODELO DE POBREZA MULTIDIMENSIONAL SEGUNDO A COMBINAÇÃO DE RECORTES DE RENDA PER CAPITA FAMILIAR E PRIVAÇÕES DE BENS E SERVIÇOS}

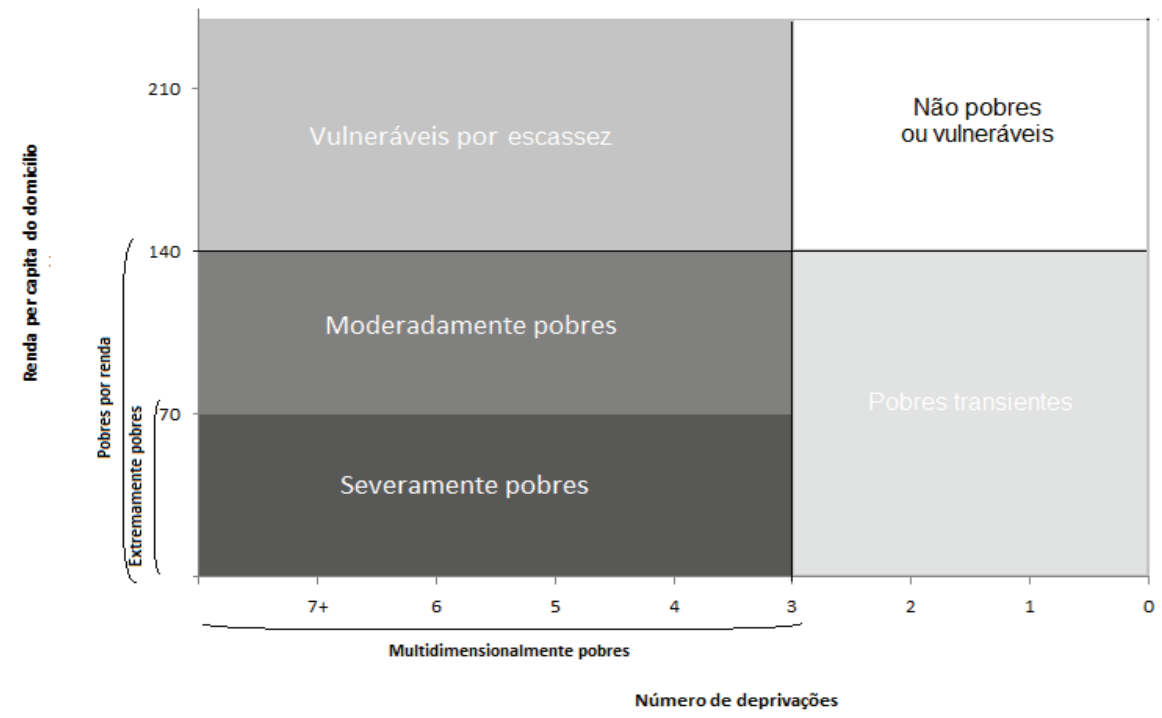

Fonte: Alkire e Foster (2011) - com adaptações dos autores

Os pobres transientes constituem-se no grupo em que a privação de necessidades básicas é menos grave que os pobres crônicos (que reúne os pobres severos e moderados), mas dispõem de renda domiciliar per capita abaixo da linha de pobreza (isto é, fazem parte da pobreza na perspectiva monetária). O termo transiente é aplicado, pois essa é uma situação em que a probabilidade de superação da pobreza é maior, dependendo, basicamente, do aumento da renda per capita.
Por fim, o grupo dos não vulneráveis indica uma situação em que o grau de vulnerabilidade é menor, seja por disporem de níveis de renda mais elevados, seja por não padecerem de privação de necessidade básicas tão intensas como os demais grupos. Estes dois grupos - transientes e não vulneráveispodem ser subdivididos conforme patamar de renda e nível de privação. Os Não Pobres seriam, pois, aqueles indivíduos com renda domiciliar acima da linha de pobreza e sem qualquer privação.

Revista Brasileira de Monitoramento e Avaliação | Número 8 | Julho-Dezembro de 2014 


\section{- QUUARO 2: TIPOLOGIA DO ÍNDICE DE POBREZA MULTIDIMENSIONAL - BANCO MUNDIAL}

\begin{tabular}{|c|c|c|c|}
\hline \multicolumn{2}{|c|}{ DIMENSÃO GERAL } & $\begin{array}{l}\text { INDICADORES } \\
\text { ESPECÍFICOS }\end{array}$ & $\begin{array}{l}\text { A PESSOA ESTÁ PRIVADA SE MORA EM DOMICÍLIO NO } \\
\text { QUAL: }\end{array}$ \\
\hline \multirow[b]{2}{*}{ Escolaridade } & D1 & Anos de escolaridade completados & Nenhum membro do domicílio completou 8 anos de estudo \\
\hline & D2 & Frequência à escola das crianças & $\begin{array}{l}\text { Pelo menos uma criança em idade escolar (7 a } 17 \text { anos) não ma- } \\
\text { triculada }\end{array}$ \\
\hline \multirow{4}{*}{ Infraestrutura } & D3 & Eletricidade & Não há acesso a energia elétrica \\
\hline & D4 & Água & Não tem rede geral com canalização interna, ou poço \\
\hline & D5 & Saneamento & Não tem rede de canalização, ou fossa séptica \\
\hline & D6 & Paredes externas & Não tem paredes externas feitas de tijolo, ou madeira tratada \\
\hline $\begin{array}{l}\text { Acesso a bens } \\
\text { e serviços }\end{array}$ & D7 & Bens & $\begin{array}{l}\text { Não tem pelo menos dois dos seguintes grupos: 1) refrigerador/ } \\
\text { freezer; 2) telefone fixo/móvel; 3) combustível limpo para cozinhar }\end{array}$ \\
\hline
\end{tabular}

Para além da importância do $\mathrm{MPI}$, outras instituições vêm também adotando indicadores multidimensionais para mensuração e compreensão do fenômeno da pobreza. O Banco Mundial, por exemplo, tem produzido importantes avanços nesse sentido, tendo já elaborado um índice nesse sentido. Na proposta de Lopez-Calva e colegas (2014), as necessidades básicas consideradas no dimensionamento da privação da pobreza multidimensional referem-se a três dimensões - educação, infraestrutura domiciliar e acesso a bens e serviços- valendo-se de sete indicadores específicos (Quadro 2).

Tendo por base esses parâmetros de avaliação, o processo de cômputo de pobres multidimensionais segue a mesma estrutura metodológica entrevista no caso do MPI da OPHI/ PNUD. Nesse mesmo sentido, também o processo de classificação final segue a mesma estrutura da Figura 1.

\section{EVOLUÇÃO DA POBREZA MULTIDIMENSIONAL NO BRASIL: 2001 A 2013}

Como discutido em estudo anterior (VAZ, 2014), as propostas de dimensionamento da pobreza na perspectiva multidimensional propõem a categorização da população em diversos grupos segundo níveis de privação de renda e necessidades básicas. A análise da série histórica do indicador multidimensional revela uma tendência de queda acentuada e sistemática ao longo do período 2001 a 2013 (Gráfico 1). Em 2001, o percentual de pobres multidimensionais era de 9,3 \% da população; já em 2013, o indicador estimava um percentual de 1,1\% da população em situação de pobreza multidimensional. Isto é, como resultado de um conjunto amplo de políticas sociais e da dinâmica do mercado de trabalho, a pobreza multidimensional caiu significativamente, retirando 14 milhões de pessoas dessa condição (de 16 milhões em 2001 para 2,2 milhões em 2013, em pobreza multidimensional). 


\section{- GRÁFICO 1: EVOLUÇÃO DA POBREZA MULTIDIMENSIONAL CRÔNICA BANCO MUNDIAL - BRASIL 2001 A 2013}

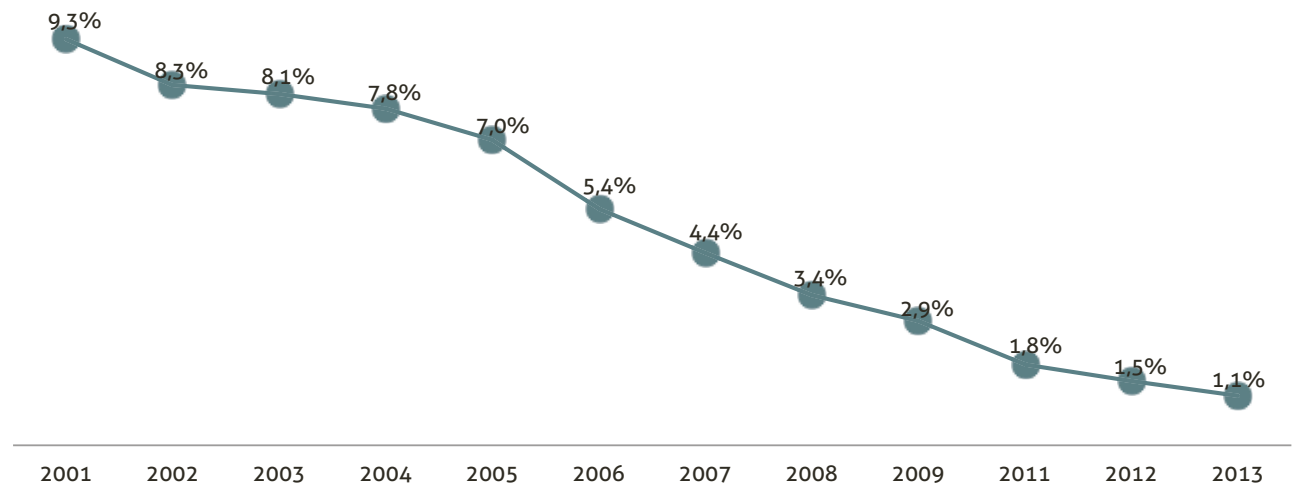

A sistematicidade da queda pode ser melhor percebida quando se observa os dados por grandes regiões (Gráfico 2), com destaque especial para o Nordeste. Ao longo do tempo há uma convergência do patamar, revelador da diminuição da desigualdade regional em termos de educação, infraestrutura domiciliar e acesso a bens no período.

Além do foco nas grandes regiões, é importante esboçar também uma análise mais detida sobre os Estados. Para tanto, optou-se por comparar dois casos que se localizam e contam com contextos e realidades socioeconômicas e demográficas historicamente diferentes, sendo o Piauí, por um lado, e Santa Catarina, por outro (Gráfico 3). Claramente, nota-se não só a queda da pobreza multidimensional nos dois estados, mas a já relatada convergência regional de patamar de pobreza, com queda muito expressiva no Piauí.

Por fim, a análise da pobreza multidimensional por raça/cor reitera a tendência já comentada de queda e convergência ao longo do período. (Gráfico 4). 
- GRÁFICO 2: EVOLUÇÃO DA POBREZA MULTIDIMENSIONAL CRÔNICA POR REGIÕES BRASIL 2001 A 2013

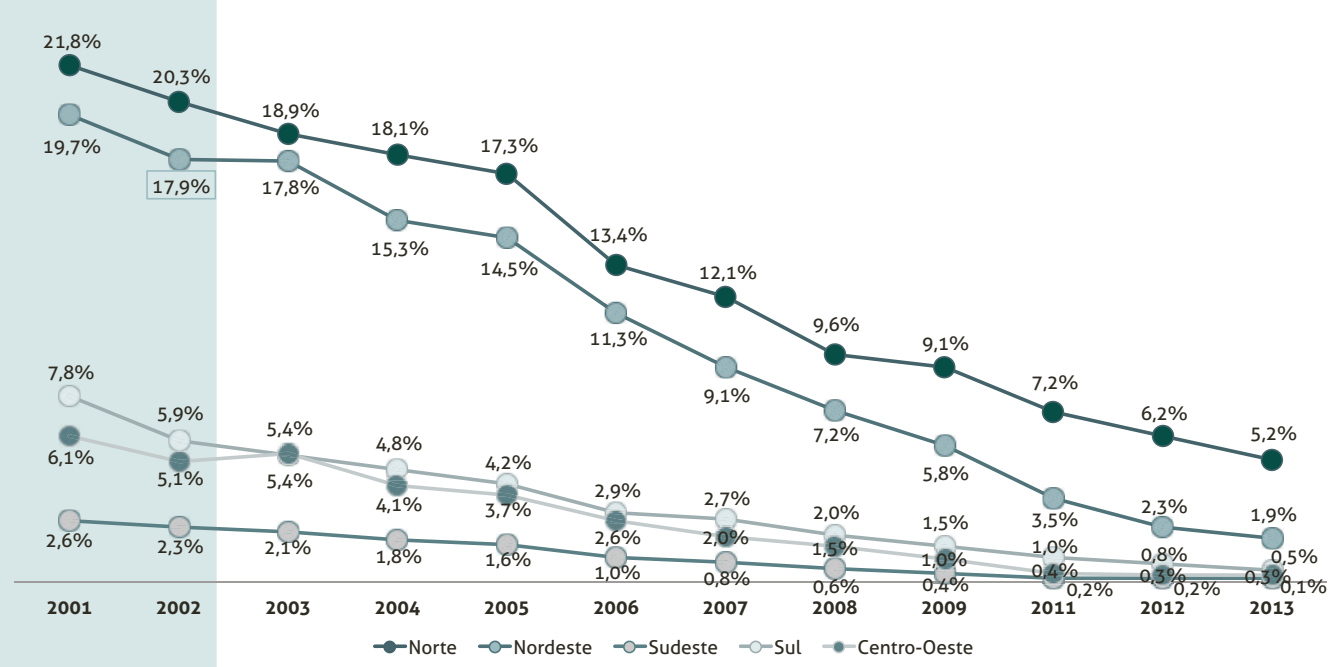

Fonte: IBGE - PNAD 2001-2013

- GRÁFICO 3: EVOLUÇÃO DA POBREZA MULTIDIMENSIONAL CRÔNICA PIAUÍ E STA CATARINA BRASIL 2001 A 2013

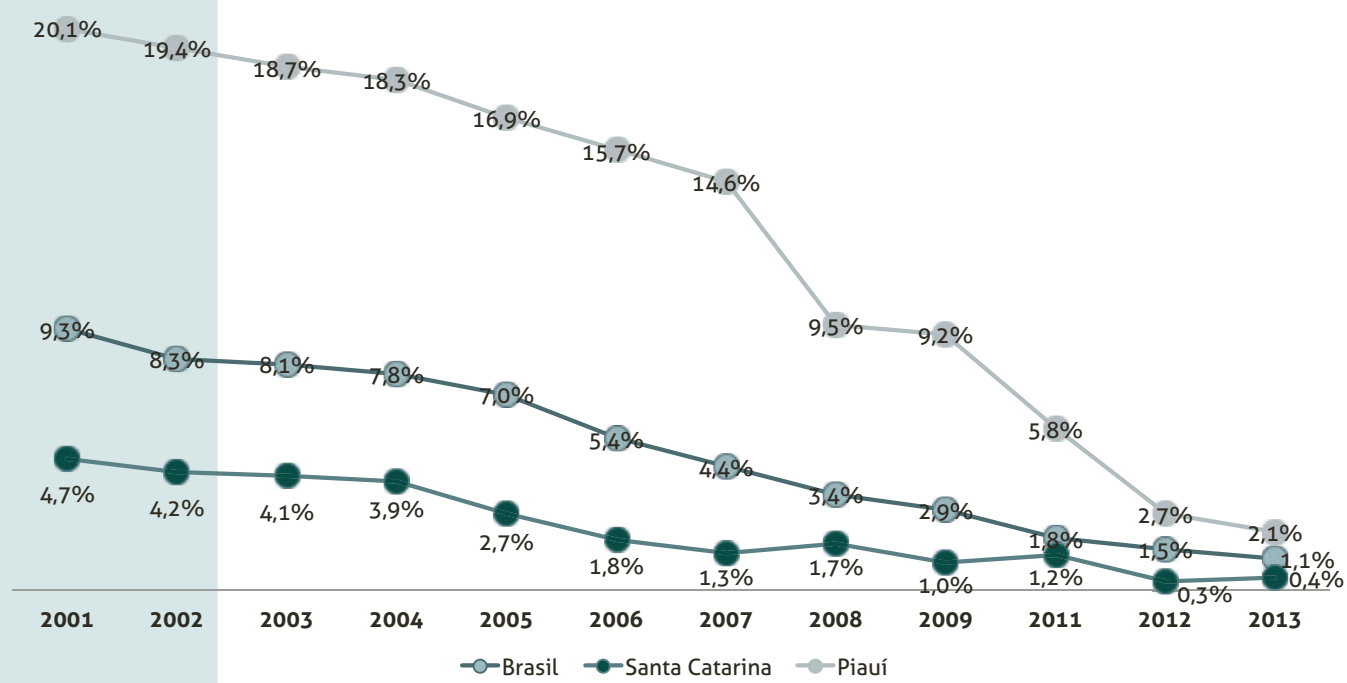

Fonte: IBGE - PNAD 2001-2013 
- GRÁFICO 4: EVOLUÇÃO DA POBREZA MULTIDIMENSIONAL CRÔNICA POR RAÇA/COR BRASIL 2001 A 2013

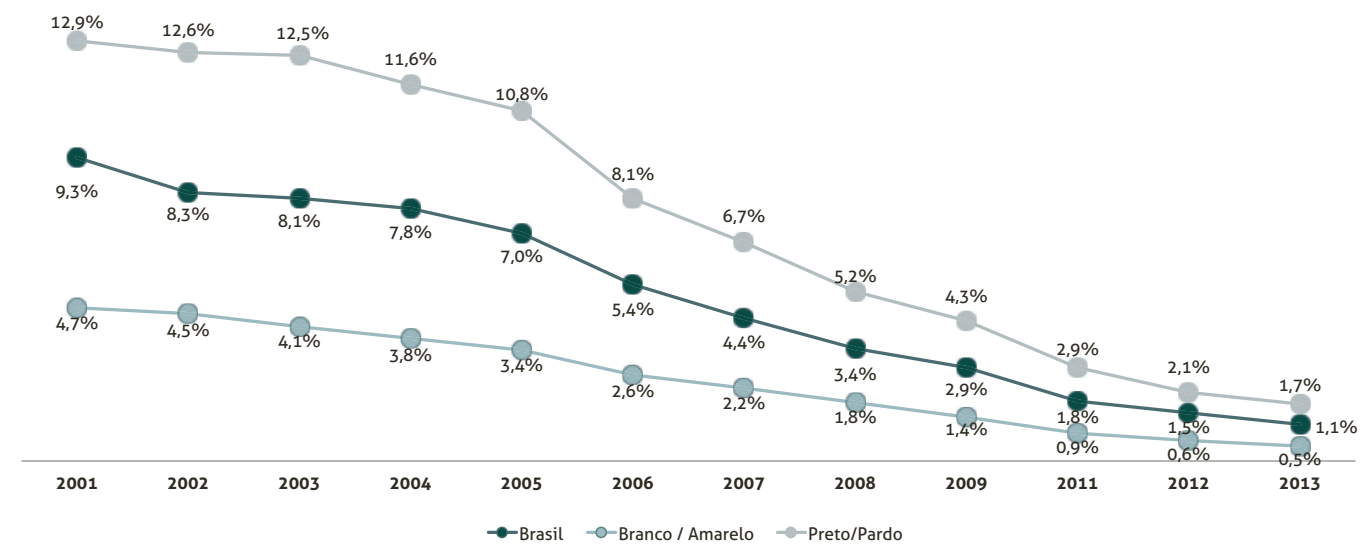

\section{AS DIFERENTES FACETAS DA POBREZA NO BRASIL EM 2013}

A multidimensionalidade da pobreza pode ser entendida também não apenas pelas diferentes dimensões em que ela pode ser apreendida mas também pelas diversas formas como ela se manifesta. De fato, como revelam diversos estudos realizados nos últimos 30 anos, os pobres compõem-se de subgrupos populacionais sujeitos a diferentes vulnerabilidades sociais no meio rural e urbano, em parte mencionados na introdução e aqui caracterizados em termos de alguns principais indicadores.

A importância de retratar os diferentes perfis sociodemográficos da pobreza multidimensional advém na necessidade de produzir subsídios para desenho de ações e programas mais específicos, voltados à mitigar as carências e iniquidades que esses grupos padecem. De fato, pela proposta de pobreza multidimensional aqui apresentada, chega-se a oito perfis diferenciados de grupos em algum grau de pobreza multidimensional, além do conjunto de "não pobres" (Tabela 1).

A Pobreza Severa e a Pobreza Moderada caracterizam-se por índices comparativamente mais elevados de informalidade, desocupação e inadequação de infraestrutura domiciliar, mais presente em famílias mais novas, com filhos, concentrando-se no Nordeste/Norte e zona rural. Perfil análogo tem os Vulneráveis, mas reunindo famílias em ciclo vital mais adiantado. Pobres Transientes apresentam-se em condição melhor nos indicadores de infraestrutura domiciliar, mas ainda com inserção ocupacional caracterizada pela informalidade, aspectos típicos da pobreza nas periferias urbanas. Entre a "mariposa" que caracteriza a pobreza severa e o "bicho da seda" dos Não pobres há graduações ilustrativas da natureza multifacetada da pobreza multidimensional (Figura 2).

Revista Brasileira de Monitoramento e Avaliação | Número 8 | Julho-Dezembro de 2014 


\section{- TABELA 1: CARACTERIZAÇÃO DOS DIFERENTES PERFIS DE POBREZA MULTIDIMENSIONAL - BRASIL 2013}

\begin{tabular}{|c|c|c|c|c|}
\hline \multirow[t]{2}{*}{$\begin{array}{l}\text { CARACTERÍSTI- } \\
\text { CA SOCIOECO- } \\
\text { NÔMICA }\end{array}$} & CATEGORIAS & $\begin{array}{c}\text { POBRES } \\
\text { TRANSIENTES } \\
\text { ATÉ } 70 \text { SEM } \\
\text { PRIVAÇÕES }\end{array}$ & $\begin{array}{c}\text { POBRES } \\
\text { TRANSIENTES } \\
\text { ATÉ } 70 \text { COM } \\
\text { ATE } 3 \text { PRIVA- } \\
\text { ÇÕES }\end{array}$ & $\begin{array}{c}\text { POBRES } \\
\text { TRANSIENTES } \\
\text { DE } 70 \text { A } 140 \\
\text { SEM PRIVA- } \\
\text { ÇÕES }\end{array}$ \\
\hline & Total de pessoas & 946.778 & 4.239 .659 & 2.643 .711 \\
\hline \multirow{3}{*}{$\begin{array}{l}\text { Sexo do Chefe de } \\
\text { Domicílio }\end{array}$} & Total de domicílios & 257.654 & 1.234 .032 & 795.833 \\
\hline & Masculino & 113.223 & 710.308 & 371.786 \\
\hline & Feminino & 144.431 & 523.724 & 424.047 \\
\hline Localizacao da & Urbana & 812.836 & 2.362 .039 & 2.360 .369 \\
\hline Residência & Rural & 133.942 & 1.877 .620 & 283.342 \\
\hline Parede do & Alvenaria ou MadeiraAparelhada & 946.778 & 3.921 .239 & 2.643 .711 \\
\hline \multirow{3}{*}{$\begin{array}{l}\text { Domicílio } \\
\text { Abastecimento de } \\
\text { Agua }\end{array}$} & Outros materiais & 0 & 295.690 & 0 \\
\hline & Rede ou PocoNascente & 803.214 & 3.044 .085 & 2.439 .334 \\
\hline & Outros & 0 & 24.199 & 0 \\
\hline Saneamento & Rede ou FossaSeptica & 757.426 & 1.630 .295 & 2.385 .638 \\
\hline \multirow{3}{*}{$\begin{array}{l}\text { Básico } \\
\text { Acesso a Energia }\end{array}$} & Outros & 0 & 1.893 .351 & 0 \\
\hline & Eletrica & 946.778 & 4.196 .735 & 2.643 .711 \\
\hline & Outros & 0 & 20.194 & 0 \\
\hline \multirow{3}{*}{$\begin{array}{l}\text { Posse de Máquina } \\
\text { de Lavar }\end{array}$} & Possui & 255.006 & 412.215 & 1.126 .114 \\
\hline & Nao possui & 691.772 & 3.804 .714 & 1.517 .597 \\
\hline & Todos os filhos tem 6 anos ou menos & 146.301 & 583.166 & 314.738 \\
\hline \multirow{4}{*}{$\begin{array}{l}\text { Tipo de Família } \\
\text { segundo ciclo de } \\
\text { vida }\end{array}$} & Todos os filhos entre 7 e 14 anos & 113.681 & 570.092 & 255.139 \\
\hline & Todos os filhos entre 15 e 24 anos & 90.045 & 299.112 & 249.624 \\
\hline & Sem filhos ou todos acima de 24 anos & 64.567 & 460.744 & 341.468 \\
\hline & Outros & 532.184 & 2.326 .545 & 1.482 .742 \\
\hline \multirow{2}{*}{$\begin{array}{l}\text { Idade do Chefe de } \\
\text { Domicílio }\end{array}$} & Chefe com 50 anos ou mais & 58.171 & 355.351 & 219.368 \\
\hline & Outros & 199.483 & 878.681 & 576.465 \\
\hline \multirow{3}{*}{$\begin{array}{l}\text { Escolaridade do } \\
\text { Chefe de Domi- } \\
\text { cílio }\end{array}$} & Sem instrucao & 28.127 & 331.037 & 53.022 \\
\hline & Ate 5 anos estudo & 59.170 & 535.558 & 132.696 \\
\hline & Outros & 170.357 & 367.437 & 610.115 \\
\hline \multirow{4}{*}{$\begin{array}{l}\text { Ocupacao das } \\
\text { pessoas de } 16 \\
\text { anos ou mais } \\
\text { Natureza do víncu- } \\
\text { lo ocupacional }\end{array}$} & Ocupado & 137.793 & 974.581 & 431.107 \\
\hline & Desocupado & 126.907 & 336.534 & 375.706 \\
\hline & Formal & 15.768 & 42.960 & 94.106 \\
\hline & Informal & 122.025 & 931.621 & 337.001 \\
\hline
\end{tabular}




\begin{tabular}{|c|c|c|c|c|c|c|}
\hline $\begin{array}{c}\text { POBRES } \\
\text { TRANSIENTES } \\
\text { DE } 70 \text { A } 140 \\
\text { COM ATE } 3 \\
\text { PRIVAÇÕES }\end{array}$ & $\begin{array}{l}\text { SEVERAMEN- } \\
\text { TE POBRES }\end{array}$ & $\begin{array}{l}\text { MODERA- } \\
\text { DAMENTE } \\
\text { POBRES }\end{array}$ & $\begin{array}{l}\text { VULNERÁ- } \\
\text { VEIS POR } \\
\text { ESCASSEZ }\end{array}$ & $\begin{array}{c}\text { NÃO POBRES } \\
\text { COM ATÉ } 3 \\
\text { PRIVAÇÕES }\end{array}$ & $\begin{array}{c}\text { NÃO POBRES } \\
\text { SEM PRIVA- } \\
\text { ÇÕES }\end{array}$ & TOTAL \\
\hline 7.015 .705 & 1.004 .372 & 1.238 .401 & 5.450 .013 & 80.501 .002 & 98.012 .331 & 201.051 .972 \\
\hline 1.723 .214 & 283.088 & 283.423 & 2.005 .416 & 27.228 .859 & 31.489 .338 & 65.300 .857 \\
\hline 1.036 .294 & 202.059 & 205.984 & 1.429 .920 & 17.225 .824 & 19.311 .983 & 40607381,00 \\
\hline 686.920 & 81.029 & 77.439 & 575.496 & 10.003 .035 & 12.177 .355 & 24.693 .476 \\
\hline 3.999 .324 & 342.271 & 403.585 & 2.304 .204 & 61.564 .264 & 96.239 .208 & 170.388 .100 \\
\hline 3.016 .381 & 662.101 & 834.816 & 3.145 .809 & 18.936 .738 & 1.773 .123 & 30.663 .872 \\
\hline 6.665 .375 & 686.398 & 992.577 & 4.675 .647 & 78.993 .435 & 98.012 .331 & 197.537 .491 \\
\hline 325.134 & 317.974 & 245.824 & 769.524 & 1.115 .544 & 0 & 3.069 .690 \\
\hline 5.526 .355 & 475.877 & 622.063 & 3.828 .509 & 74.454.119 & 97.196 .011 & 188.389 .567 \\
\hline 27.225 & 35.095 & 38.572 & 207.250 & 291.958 & 0 & 624.299 \\
\hline 2.837 .210 & 154.904 & 236.030 & 1.257 .336 & 43.227 .072 & 97.222 .889 & 149.708 .800 \\
\hline 3.348 .016 & 593.380 & 848.048 & 3.734 .003 & 34.945 .157 & 0 & 45.361 .955 \\
\hline 6.964 .205 & 853.221 & 1.080 .377 & 4.995 .957 & 80.036 .375 & 98.012 .331 & 199.729 .690 \\
\hline 26.304 & 151.151 & 158.024 & 449.214 & 72.604 & 0 & 877.491 \\
\hline 1.206 .549 & 64.373 & 106.704 & 1.030 .238 & 36.118 .012 & 75.543 .111 & 115.862 .322 \\
\hline 5.783 .960 & 939.999 & 1.131 .697 & 4.414 .933 & 43.990 .967 & 22.469 .220 & 84.744 .859 \\
\hline 745.296 & 130.188 & 143.194 & 386.951 & 8.089 .367 & 11.923 .987 & 22.463 .188 \\
\hline 854.325 & 89.867 & 143.757 & 669.664 & 10.493 .084 & 12.379 .863 & 25.569 .472 \\
\hline 454.327 & 49.679 & 48.805 & 619.658 & 12.914 .176 & 20.488 .886 & 35.214 .312 \\
\hline 469.402 & 133.368 & 81.584 & 1.898 .231 & 22.070 .109 & 25.251 .919 & 50.771 .392 \\
\hline 4.492 .355 & 601.270 & 821.061 & 1.875 .509 & 26.934 .266 & 27.967 .676 & 67.033 .608 \\
\hline 453.616 & 74.581 & 74.638 & 1.236 .575 & 14.014 .849 & 13.371 .740 & 29.858 .889 \\
\hline 1.269 .598 & 208.507 & 208.785 & 768.841 & 13.214 .010 & 18.117 .598 & 35.441 .968 \\
\hline 387.114 & 111.994 & 92.476 & 806.462 & 5.359 .446 & 1.489 .446 & 8.659 .124 \\
\hline 740.071 & 131.591 & 154.964 & 991.678 & 10.581 .867 & 4.717 .527 & 18.045 .122 \\
\hline 596.029 & 39.503 & 35.983 & 207.276 & 11.287 .546 & 25.282 .365 & 38.596 .611 \\
\hline 2.035 .009 & 331.566 & 452.760 & 2.584 .255 & 37.943 .294 & 51.448 .498 & 96.338 .863 \\
\hline 384.836 & 52.700 & 24.546 & 73.350 & 2.071 .409 & 3.279 .337 & 6.725 .325 \\
\hline 198.237 & 10.842 & 27.245 & 584.576 & 19.514 .282 & 38.295 .845 & 58.783 .861 \\
\hline 1.836 .772 & 320.724 & 425.515 & 1.999 .679 & 18.429 .012 & 13.152 .653 & 37.555 .002 \\
\hline
\end{tabular}

Revista Brasileira de Monitoramento e Avaliação | Número 8 | Julho-Dezembro de 2014 
- FIGURA 2: PERFIS DA POBREZA MULTIDIMENSIONAL EM 2013

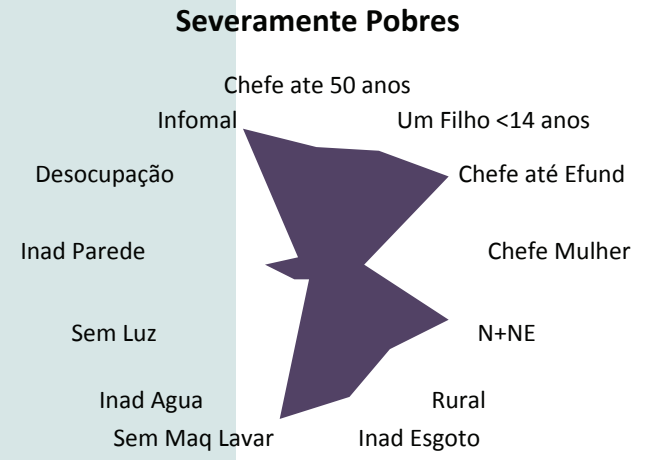

Moderadamente Pobres

Chefe ate 50 anos

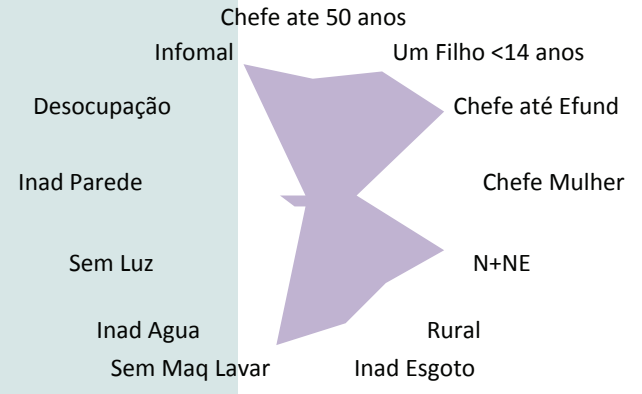

\section{Vulneráveis por escassez}

Chefe ate 50 anos

Infomal Um Filho $<14$ anos

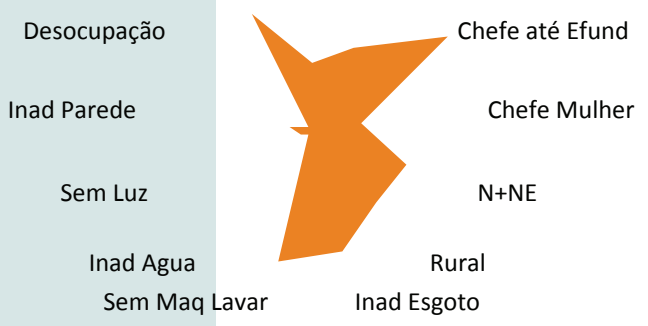

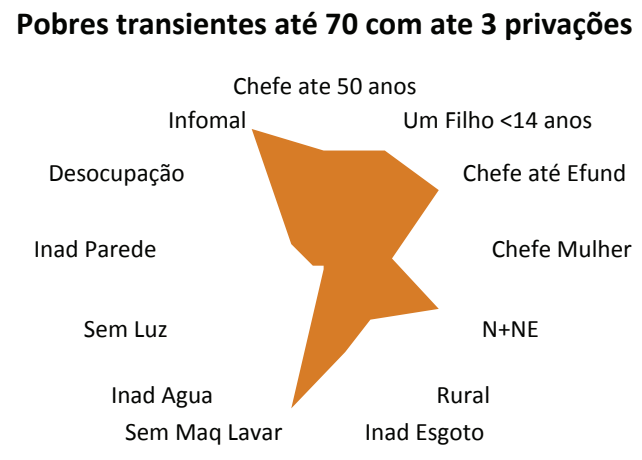

Pobres transientes de 70 a 140 com ate 3 privações

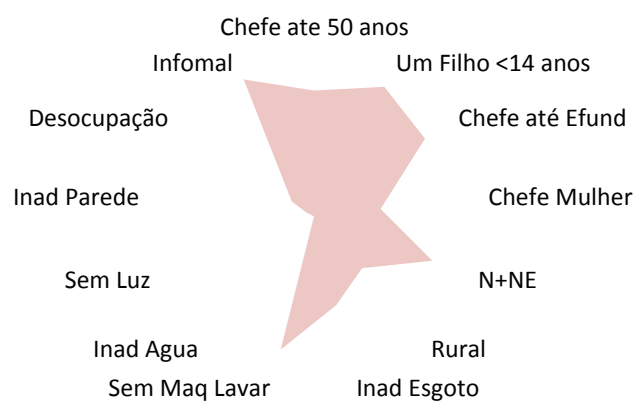

Náo pobres com até 3 privações

\author{
Chefe ate 50 anos \\ Infomal Um Filho $<14$ anos
}

Desocupação

Chefe até Efund

Inad Parede

Sem Luz

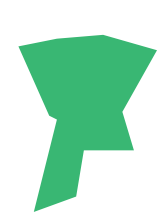

Chefe Mulher

$\mathrm{N}+\mathrm{NE}$

Inad Agua

Rural

Sem Maq Lavar Inad Esgoto 


\section{Pobres transientes até 70 sem privações}

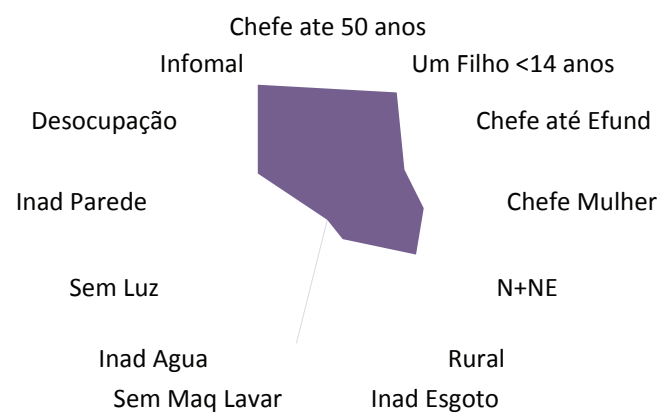

\section{Pobres transientes de 70 a 140 sem privações}

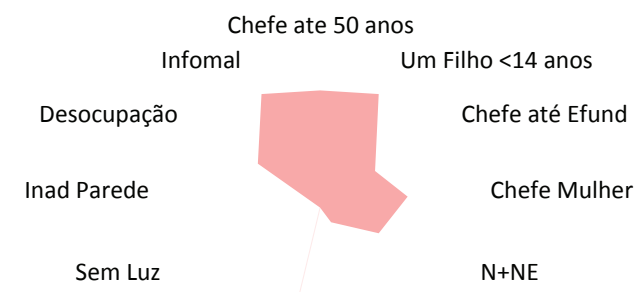

Inad Agua

Sem Maq Lavar

Rural

\section{Não pobres sem privações}

\begin{tabular}{|c|c|}
\hline \multicolumn{2}{|c|}{ Chefe ate 50 anos } \\
\hline Infomal & Um Filho $<14$ anos \\
\hline Desocupação & Chefe até Efund \\
\hline Inad Parede & Chefe Mulher \\
\hline Sem Luz & $\mathrm{N}+\mathrm{NE}$ \\
\hline Inad Agua & Rural \\
\hline Sem Maq Lavar & lad Esgoto \\
\hline
\end{tabular}

\section{Considerações finais}

Para finalizar, vale reiterar parte das considerações expostas no Estudo Técnico 08/2014 acerca da mensuração multidimensional da pobreza. Ainda que o indicador de pobreza multidimensional aqui apresentado consiga captar parte significativa dos esforços multissetoriais de políticas e programas que o Brasil vem realizando na superação da pobreza, há ainda avanços conceituais e operacionais que poderiam e deveriam ser efeitos.

Sob sua configuração original, o indicador não contempla dimensões importantes e cruciais da determinação da Pobreza (em sentido multidimensional) como acesso a Trabalho e à Proteção Social. No que tange

Revista Brasileira de Monitoramento e Avaliação | Número 8 | Julho-Dezembro de 2014 
especificamente a temática do mercado de trabalho o índice não contempla indicadores sobre a participação relativa dos chamados "nem-nem" (população que não trabalha nem estuda) entre os jovens, percentual de informalidade na ocupação e, por fim, sequer taxa de ocupação da PIA ou até mesmo da força de trabalho primária (população de 25 a 59 anos). O acesso a emprego e renda é uma dimensão que impacta diretamente na constituição ou não de privações que caracterizam a pobreza. No contexto brasileiro, aos avanços na geração de empregos, em especial, empregos formais combinados com uma política de valorização do salário mínimo não estão refletido diretamente no cômputo do índice.

Aspectos relacionados a proteção social como trabalho infantil e idosos sem remuneração seja pelo trabalho, seja por instituto oficial de previdência, são privações que também não são consideradas no índice. Dispor ou não de Aposentadoria, Benefício de Prestação Continuada ou dos benefícios do Programa Bolsa Família são aspectos cruciais a que as famílias estão sujeitas no país.

Ademais, mesmo em dimensões consideradas, como Educação, na configuração atual do indicador, não se capta acesso a creche, aspecto que para além de contribuir com a escolarização da população, cria condições para propiciar aos pais tempo e disposição para ingresso no mercado de trabalho e para a qualificação profissional.
Em relação a dimensão de Bens e Serviços o indicador define combinações entre posse de veículos de transporte e acesso a eletrodomésticos deixando de captar importantes avanços que o país vem pautando especialmente na última década no que tange ao acesso a bens pela população de uma forma geral. Além disso, a metodologia não diferencia as zonas urbanas e rurais. $\mathrm{O}$ conceito de adequado para indicadores que compõem esta dimensão devem ser relativizados em função das diferenças que marcam esses contextos. Por exemplo, no caso do acesso à água, tanto para os domicílios urbanos quanto rurais considerou-se que o domicílio estaria em privação se não tivesse acesso à rede geral de distribuição com canalização interna; sendo que na zona rural, acesso a poço e/ou nascente com canalização interna é atributo de adequação no acesso à água. Com relação à privação de mobilidade, importa mais saber se a população tem acesso a transporte público próximo à residência do que automóvel ou motorcicleta.

Por fim, a incorporação de outras dimensões e variáveis na medição de um indicador de pobreza multidimensional pode trazer uma contribuição interessante para avaliar o efeito integrado de várias políticas e programas sociais no país, sejam eles de natureza universal, sejam eles voltados à população mais vulnerável. A discussão dos indicadores da agenda de desenvolvimento pós-2015 e o estabelecimento de um nova pauta de prioridades em termos de política social no próximo ano no Brasil é um boa oportunidade para discussão desse novo indicador. 


\section{Referências bibliográficas}

ALKIRE, S.; FOSTER,J. Counting and multidimensional poverty measurement. Journal of Public Economics, v. 95, n. 7, p. 476- 487, 2011.

CONEVAL. Informe de Pobreza Multidimensional em Mexico, 2008. Disponível em: <http:// www.coneval.gob.mx/rw/resource/coneval/ info_public/PDF_PUBLICACIONES/INFORME_ POBREZA_MULTI_WEB.pdf $>$. Acesso em: $04 \mathrm{dez}$. 2014.

CORTÉS, Fernando. Desigualdad económica y poder. RELATÓRIO CEPAL, 2010. Disponível em: <http://www10.iadb.org/intal/intalcdi/ PE/2010/07543.pdf>. Acesso em: 04 dez. 2014.

FERES, J. C.; VILLATORO; Pablo. Cadernos de Estudos Desenvolvimento Social em Debate. A viabilidade de se erradicar a pobreza: uma análise conceitual e metodológica. Brasília: SAGI, 2013.

FOSTER, J., GREER, J., THORBECKE, E.: A class of decomposable poverty measures. Econometrica, v. 52, p. 761-776, 1984.

JANNUZZI, Paulo de Martino ; MARTIGNONI, Enrico Moreira ; SOUTO, B. F. O Programa Bolsa Família e sua contribuição para redução da pobreza no Brasil. Revista Brasileira de Monitoramento e Avaliação, v. 4, p. 40-61, 2012.
LOPEZ-CALVA, L.; LACH, S; FRUTTERO, A. Medindo pobreza crônica no Brasil. In: BRASIL. Ministério do Desenvolvimento Social e Combate à Fome. Cadernos de Estudos Desenvolvimento Social em Debate. Brasília, n.22 (no prelo).

SEN, A. Commodities and Capabilities. Oxford: Oxford University Press, 1985.

A sociological approach to the measurement of poverty: a reply to Professor Peter Townsend. Oxford Economic Papers, New Series, v. 37, p. 669-676, 1985.

SOARES, S. A distribuição dos rendimentos do trabalho e a queda da desigualdade de 1995 a 2009. Mercado de Trabalho. Brasília: IPEA, número 45, pp. 35-40, 2010.

VAZ, Alexander Cambraia N. Notas críticas conceituais e metodológicas referentes ao IPS - Índice de Progresso Social. Estudo Técnico SAGI n' 18/2013. Disponível em: <www.mds.gov.br/ sagi>. Acesso em: 04 dez. 2014.

VAZ, Alexander Cambraia N. Pobreza Multidimensional. Estudo Técnico SAGI nº 08/2014. Disponível em: <www.mds.gov.br/sagi>. Acesso em: 04 dez. 2014.

Revista Brasileira de Monitoramento e Avaliação | Número 8 | Julho-Dezembro de 2014 